\title{
Phenotypic Characterization and Risk Factors of Nosocomial Staphylococcus aureus from Health Care Centers
}

\author{
Chandrashekhar G. Unakal $^{1 *}$, Basappa B. Kaliwal ${ }^{2}$ \\ ${ }^{1}$ Deptartment of Microbiology, University of Gondar, Gondar, Ethiopia \\ ${ }^{2}$ P.G. Department of Studies in Biotechnology and Microbiology, Karnatak University, Dharwad, India \\ Email: "cg.unakal@gmail.com
}

Received April 7, 2012; revised April 25, 2012; accepted May 8, 2012

\begin{abstract}
Multidrug resistant Staphylococcus aureus (MDRS) is a serious threat to hospitalized patients globally and now represents a challenge for public health, as community-acquired infections appear to be on the increase in both adults and children. S. aureus colonization has been shown to be a risk factor for community-acquired and nosocomial infections. A total of 130 subjects from the community and 100 subjects from health care-related facilities were evaluated for the prevalence of Staphylococcus aureus colonization and to identify risk factors associated with methicillin-resistant $S$. aureus (MRSA) and vancomycin resistant S. aureus (VRSA) colonization. Among the community subjects, $35.38 \%$ had MRSA and $1.53 \%$ VRSA colonization. Subjects from health care-related facilities had a lower MRSA colonization rate $(17 \%)$ than community subjects and the colonization VRSA has not been found. Age was a risk factor for S. aureus colonization, with subjects under age 20 years or between 60 and 80 years showing higher rates of colonization. In conclusion, a high prevalence of MRSA colonization was observed among people with relationship to the hospital setting. The high level of multiple-drug resistance among community MRSA strains in association with the previously reported excessive use of antibiotics highlights the importance of the problem of antibiotic selective pressure. Our results indicate that the spread of both MRSA and VRSA and the transmission of hospital isolates contribute to the high MRSA/ VRSA burden in the community.
\end{abstract}

Keywords: Staphylococcus aureus; Nosocomial; MRSA; VRSA

\section{Introduction}

In the past few decades, methicillin-resistant Staphylococcus aureus (MRSA) has been recognized as an important nosocomial pathogen worldwide [1]. The emergence and rapid spread of this organism has created important new challenges for infection prevention and control services in hospitals and other health care facilities. Interestingly, there appears to be significant variability in the epidemiology and prevalence of MRSA in different parts of the world and even in different regions of a country [2].

Patients in the intensive care units (ICU) are at a higher risk of acquiring nosocomial infections compared with patients in general wards [3]. This is partly because of the severity of the underlying illnesses and partly because of iatrogenic factors related to the high frequency of invasive procedures required for monitoring and treatment [4]. Bacteremia continues to be a major cause

${ }^{*}$ Corresponding author. of morbidity and mortality in hospitals [5]. The overall or crude rate of death does not distinguish between the contribution of the patients' underlying diseases and the contribution of bloodstream infections [6]. The prognosis of post-bacteremia infection (true bacteremia or fungaemia) is very variable.

The widespread use of these antibiotics in the 1950s induced the predominance of b-lactamase-producing resistant strains. To solve the problem, the b-lactamaseresistant penicillins were developed, but reports of resistance to this new group started to appear in the 1960s in Europe and in the 1970s in the United States [7]. The emergence of antibiotic-resistant strains of $S$. aureus is now considered to be a major problem in most hospitals. Virtually, all nosocomial strains produce a b-lactamase and thus are resistant to penicillins. Moreover, data from the Centers for Disease Control and Prevention indicate that throughout the United States there has been an increase in the frequency of methicillin-resistant $S$. aureus (MRSA) strains resistant to multiple antibiotics in both 
large and small hospitals [8]. Thus far, all strains of MRSA have been susceptible to vancomycin, although certain strains have exhibited tolerance. It is possible, however, that vancomycin resistant grampositive cocci such as Enterococcus spp. and Staphylococcus haemolyticus may transmit the gene (s) responsible for this phenotype to $S$. aureus, leaving few if any options for antimicrobial chemotherapy of infections caused by the organisms [9].

Recent studies suggest that the infection due to MRSA is not only hospital-acquired but community acquired as well [10]. Some large outbreaks have been reported from different parts of the world, where it had caused severe infections including septicemia, endocarditis and meningitis [11]. A study by Dickinson in England and Wales has concluded an increase in the trend of death due to MRSA infection [12]. Infections caused by MRSA can be expensive in terms of antibiotic therapy, isolation facilities and materials and length of hospital stay. According to a World Health Organization literature, the global financial burden because of MRSA infection has been worked out to be $\$ 20,000$ to $\$ 114,000$ for outbreaks and from $\$ 28,000$ to $\$ 1600,000$ for endemic infections per year. The common sources of these infections are human patients and carriers [13]. The risk factors that contribute to MRSA are antibiotics abuse, prolonged hospitalization, intravascular instrumentation and hospitalization in an intensive care unit [14]. There is considerable variation in numbers of clinical infections among units, hospitals and countries.

The analyses of the data showed a higher prevalence of $S$. aureus in nursing staff and attendants compared to the doctors. Age, sex, health status could not be correlated with the rate of infection; however, it could be due to the nature of job and place of work of the individuals. The prevalence of $S$. aureus was found higher in surgical wards than the general wards. Our study shows that the risk of infection is higher in individuals occupationally exposed to such microbes. MRSA has been reported earlier from hospitals in various parts of world [15]. There is a need to screen individuals in hospitals for risk exposures and infections, to avoid outbreak and cross infections. Other factors including prolonged hospitalization, multiple antibiotic therapy sessions, and intravenous catheterization also increase the risk of nosocomial infections in burn patients. MRSA is an important causative agent of nosocomial infections in India. According to an Indian study, the prevalence of infections caused by MRSA has increased from 12 percent in 1992 to 80.03 percent in 1999 [16]. Many of these MRSA isolates are becoming multidrug-resistant, and are susceptible only to glycopeptides [17].

In the $1980 \mathrm{~s}$, due to the widespread occurrence of MRSA, empiric therapy for staphylococcal infections (particularly nosocomial sepsis) was changed to vancomycin in many health-care institutions. Vancomycin use in many countries also increased during this period because of the growing numbers of infections with Clostridium difficile and coagulase-negative staphylococci in health-care facilities [17]. Thus, the early 1990s saw a discernible increase in vancomycin use. As a consequence, selective pressure was established that eventually led to the emergence of strains of Staphylococcus aureus and other species of staphylococci with decreased susceptibility to vancomycin, but in 1997 the first clinical isolate of Staphylococcus aureus with reduced susceptibility to vancomycin was reported from Japan [18]. Data from the December 2000 report of the National Nosocomial Infection Surveillance (NNIS) System indicated that about $75 \%$ of coagulase-negative staphylococci and $47 \%$ of $S$. aureus isolates from intensive care units were resistant to methicillin (www.cdc.gov/ncidod/hip/NNIS/DEC2000sar. PDF). Vancomycin remains the drug of choice for these infections. Vancomycin resistance among staphylococci was developed in laboratories even before the drug was in use clinically [19]. However, this resistance was so difficult to induce that many felt it would be unlikely to occur in a clinical setting [20]. That no vancomycin-resistant staphylococci were reported in the first 20 years the drug was used only strengthened this assumption. Unfortunately, this confidence was shattered by the first reports of vancomycin resistance in coagulase-negative staphylococci in 1979 and 1983 [21]. In our previous study it has been reported that the VRSA strain (MIC 32 $\mu \mathrm{g} / \mathrm{ml}$ ) has been isolated from the CSF of a 14 year old boy admitted in hospital having diagnosed for meningitis, 6 VRSA strains were isolated from pus of post operative wound infection and burnt skin. Other 7 VISA (MIC 8 $\mu \mathrm{g} / \mathrm{ml}$ ) strains were isolated from amputated wound and diabetic foot lesions [22]. The present study was carried out to determine the risk factors for nosocomial acquisition of MRSA/VRSA, phenotypic characterization of the Staphylococcus aureus and mortality in health care centers.

\section{Materials and Methods}

\subsection{Study Duration and Population}

This study was carried out at the Department of Microbiology and Biotechnology Karnatak University, Dharwad. The samples were obtained from the personals served as subjects of various Nursing Homes, Hospitals and other Health Care Centers of Hubli-Dharwad. Nasal swabs from both anterior nares were obtained from healthy subjects working in health care centers of Hubli-Dharwad. Samples were also taken from healthy volunteers including adults of both sexes residing the hospital premises. Any person with a history of Hospitalization, un- 
dergoing surgery or any kind of treatment and intake of antibiotics in the past three months was ruled out of the study.

\subsection{Microbiological Study}

All study participants underwent swabbing of the anterior $1.5 \mathrm{~cm}$ of the nasal vestibule of both nares with a sterile swab. The swab specimen was streaked onto two mannitol salt agar plates (Hi-Media, Mumbai) one of which was supplemented with oxacillin $(6 \mu \mathrm{g} / \mathrm{ml})$. These inoculated plates were incubated at $37^{\circ} \mathrm{C}$ for $48 \mathrm{~h}$, after which morphological and Gram stain examinations were conducted. Colonies of interest were selected for further inoculation onto Brain Heart Infusion agar plates (HiMedia, Mumbai.) at $37^{\circ} \mathrm{C}$ overnight. The coagulase test was used to identify S. aureus. Methicillin-susceptible $S$. aureus (MSSA) was preliminarily detected by its characteristic growth on mannitol salt agar and the absence of growth in the presence of oxacillin, while growth on both agar plates was presumed to indicate the presence of MRSA. All isolates were inoculated onto Mueller-Hinton agar (Hi-Media, Mumbai) containing $6 \mu \mathrm{g}$ of oxacillin per $\mathrm{ml}$ and $4 \% \mathrm{NaCl}$ to confirm methicillin resistance.

\subsection{Catalase Test}

The catalase test was done by transferring a small portion of the culture with a clean glass rod onto a slide with $3 \%$ $(\mathrm{v} / \mathrm{v})$ hydrogen peroxide $\left(\mathrm{H}_{2} \mathrm{O}_{2}\right)$ which is kept under cover of a Petri plate to avoid aerosols. If the bacteria produce, they will split hydrogen peroxide and oxygen will be evolved. The evolution of gas causes bubbles to form and is indicative of a positive test.

\subsection{Oxidation Fermentation Test}

Bacteria were inoculated into two oxidation fermentation tubes, $\mathrm{n}$ one tube sterile liquid paraffin was added to provide anaerobic condition, and the tubes were incubated at $37^{\circ} \mathrm{C}$. The tubes were observed for colour change indicating oxidative and fermentative breakdown of the sugars and the observations recorded.

\subsection{Mannitol Fermentation}

To perform this test Mannitol salt agar (Hi-Media) was used which contains D-Mannitol and phenol red as $\mathrm{pH}-$ indicator. The MSA plates were inoculated with test organism and incubated at $37^{\circ} \mathrm{C}$ for $24 \mathrm{~h}$. The colony characters and colour change was recorded.

\subsection{Coagulase Test}

A single colony of overnight cultures on Trypticase soya agar (TSA) was used for a coagulase test following the conventional protocol except that the pre-incubation times in brain heart infusion (BHI) prior to the enzyme assay was $24 \mathrm{~h}$. The BHI broth was used for the coagulase. The formation of a clot was examined at 2, 4, 6, and $24 \mathrm{~h}$.

\subsection{Antibacterial Susceptibility Testing}

Antibiotic susceptibility screening was done as per the guidelines of National Committee for Clinical Laboratory Standards (NCCLS). Kirby-Bauer's disc diffusion technique was adapted for antibiogram [23]. The antibiotic discs and Mueller-Hinton Agar purchased from HiMedia, Mumbai. The plates were prepared as per the manufacturer's instructions and checked for sterility by incubating the plates overnight at $37^{\circ} \mathrm{C}$. The antibiotics used in this study were obtained from Hi-Media, Mumbai. The antibiotics discs were kept at room temperature for 1 hour. The agar plates were overlaid with inoculums of Staphylococcus aureus showing the turbidity equivalent to that of a 0.5 McFarland standard.

In this study methicillin susceptible S. aureus ATCC 29213, methicillin resistant S. aureus ATCC 43300 were used as reference strains.

\section{Results}

A total of 230 samples were collected from various health care centers including males and females of the age 18 to 80 years. Of the total the samples were collected from 149 male and 81 female individuals (Table 1). Locations for the sample collection were both the anterior nares, forearm and dorsum of palm. A total of $200(87 \%)$ Staphylococcus isolates were obtained (142 males, 58 females) (Table 2) and 130 (57\%) Staphylococcus aureus were confirmed based on staining character, growth on mannitol salt agar, coagulase test and other biochemical parameters (Table 3). The results for disc diffusion test using 14 antibiotics for 130 isolates of Staphylococcus aureus are shown in Table 4. Disc diffusion test revealed that all the isolated strains were resistant $(100 \%)$ to penicillin, where as cloxacillin, oxacillin and methicillin were shown the resistance $97.42 \%$, $35.90 \%$, and $35.38 \%$ respectively. $72.66 \%, 84.11 \%$, $73.01 \%$ of the total isolates have showed resistance to tetracycline, erythromycin and rifampicin respectively. Whereas ciprofloxacin, gentamycin, streptomycin and kannamycin resistance in Staphylococcus aureus was $67.75 \%, 86.5 \%, 95.75 \%$ and $94.97 \%$ respectively. Amphicillin, ceftriaxone and vancomycin have also showed $86.68 \%, 85.51 \%$, and $1.53 \%$ resistance respectively. Many of the MRSA strains were resistant to all the tested antibiotics. The prevalence of MRSA, MSSA and VRSA from the isolates showed $35.38 \%, 64.62 \%$ and $1.53 \%$ respectively (Table 5). 
Table 1. Number of individuals screened for Staphylococcus isolates.

\begin{tabular}{|c|c|c|c|c|c|}
\hline \multirow{2}{*}{ Location } & \multirow{2}{*}{ Population Group } & \multirow{2}{*}{ Age Group } & \multicolumn{3}{|c|}{ Total No. of Individuals } \\
\hline & & & Male & Female & Total \\
\hline Nursing Home & Nurses, Staff & $18-30$ Yrs & 54 & 26 & 80 \\
\hline Hospital & Nurses, Staff & $18-50$ Yrs & 32 & 28 & 60 \\
\hline Private Clinic & Staff & $18-60$ Yrs & 25 & 15 & 40 \\
\hline Medical College & Duty Doctors Staff & $20-80$ Yrs & 38 & 12 & 50 \\
\hline Total & & & 149 & 81 & 230 \\
\hline
\end{tabular}

Table 2. Location wise distribution of Staphylococcus isolates.

\begin{tabular}{|c|c|c|c|c|c|c|c|c|c|c|}
\hline \multirow{2}{*}{ Location } & \multirow{2}{*}{ Isolate } & \multicolumn{2}{|c|}{ Anterior Nares } & \multicolumn{2}{|c|}{ Forearm } & \multicolumn{2}{|c|}{ Dorsum of palm } & \multicolumn{2}{|c|}{ Total } & \multirow[t]{2}{*}{ Total } \\
\hline & & $\mathbf{M}$ & $\mathbf{F}$ & $\mathbf{M}$ & $\mathbf{F}$ & $\mathbf{M}$ & $\mathbf{F}$ & $\mathbf{M}$ & $\mathbf{F}$ & \\
\hline Nursing Home & Staphylococci & 14 & 04 & 20 & 08 & 10 & 04 & 44 & 16 & 60 \\
\hline Hospital & Staphylococci & 16 & 08 & 10 & 10 & 06 & - & 32 & 18 & 50 \\
\hline Private Clinic & Staphylococci & 11 & 02 & 06 & 02 & 03 & 01 & 20 & 05 & 25 \\
\hline Medical College & Staphylococci & 10 & 09 & 22 & 08 & 14 & 02 & 46 & 19 & 65 \\
\hline Total & & & & & & & & 142 & 58 & 200 \\
\hline
\end{tabular}

Table 3. Proportion of Staphylococcus aureus among the total staphylococci isolated from different location.

\begin{tabular}{|c|c|c|c|c|c|c|c|c|c|}
\hline \multirow{2}{*}{ Location } & \multirow{2}{*}{ Isolate } & \multicolumn{2}{|c|}{ Anterior Nares } & \multicolumn{2}{|c|}{ Forearm } & \multicolumn{2}{|c|}{ Dorsum of palm } & \multicolumn{2}{|c|}{ Total $(n=130)$} \\
\hline & & $\mathbf{M}$ & $\mathbf{F}$ & $\mathbf{M}$ & $\mathbf{F}$ & $\mathbf{M}$ & $\mathbf{F}$ & $\mathbf{M}$ & $\mathbf{F}$ \\
\hline Nursing Home & Staphylococcus aureus (\%) & 40 & 6.7 & 33.3 & 10 & 6.7 & 3.3 & 80 & 20 \\
\hline Hospital & Staphylococcus aureus (\%) & 25 & 20 & 20 & 25 & 10 & - & 55 & 45 \\
\hline Private Clinic & Staphylococcus aureus (\%) & 30 & 10 & 30 & 10 & 15 & 5 & 75 & 25 \\
\hline Medical College & Staphylococcus aureus (\%) & 25 & 10 & 25 & 7.5 & 27.5 & 5 & 77.5 & 22.5 \\
\hline Total & & & & & & & & 70.77 & 29.23 \\
\hline
\end{tabular}

Table 4. Resistant pattern of various antimicrobial agents determined by disc diffusion method.

\begin{tabular}{cccc}
\hline Antibiotics & No. of Resistant strains (\%) & No. of Intermediate strains (\%) & No. of Sensitive strains (\%) \\
\hline Penicillin & 100 & - & - \\
Cloxacillin & 97.42 & 2.58 & - \\
Oxacillin & 54.90 & 30.1 & 15 \\
Methicillin & 35.38 & - & 64.62 \\
Tetracycline & 72.66 & 20.34 & 7 \\
Erythromycin & 84.11 & 14 & 1.99 \\
Refampicin & 73.01 & 26.99 & - \\
Ciprofloxacin & 67.75 & 20 & 12.25 \\
Gentamicin & 86.5 & 10 & 3.5 \\
Streptomycin & 95.75 & 4.25 & - \\
Kannamycin & 94.97 & 5.03 & - \\
Ampicillin & 86.68 & 10 & 3.32 \\
Ceftriaxone & 85.51 & 10.49 & 4 \\
Vancomycin & 1.5 & 4.10 & 94.85 \\
\hline
\end{tabular}


Table 5. Prevalence of MRSA/MSSA and VRSA/VISA from Staphylococcus aureus isolates.

\begin{tabular}{ccc}
\hline S. aureus Isolated $(\mathbf{n}=\mathbf{1 3 0})$ & No. of isolates & Percentage \\
\hline MRSA & 46 & 35.38 \\
MSSA & 84 & 64.62 \\
VRSA & 02 & 1.53 \\
VISA & - & - \\
\hline
\end{tabular}

MRSA: Methicillin Resistant S. aureus; MSSA: Methicillin Susceptible $S$. aureus; VRSA: Vancomycin Resistant S. aureus; VISA: Vancomycin Intermediate Susceptible S. aureus.

\section{Discussion}

S. aureus, antibiotic-resistant Gram-negative bacteria, and Candida spp. are among the pathogens responsible for bloodstream infections, which are usually associated with the poorest outcomes [24]. In recent years, the Staphylococcus aureus causing nosocomial infections has increased because of their ability to express certain resistance phenotypes [25] and hence they continue to be one of the major sources of morbidity and mortality [26]. While in these studies the most frequently isolated pathogens that caused NB infections were Klebsiella spp., Candida spp., Enterococcus spp. and P. aeruginosa, in our study we concentrated on, methicillin-resistant $S$. aureus, as a source of secondary bacteraemia, has been reported to be more frequent among patients [27]. However, primary bloodstream infection, in which no source could be determined, was also reported to be the most frequent source of NB [28]. Broad-spectrum antibiotic use was found to be the most important risk factor associated with the occurrence of this undesirable medical practice. Nosocomial infection, nosocomial pneumonia, older age, mechanical ventilation, enteral nutrition, tracheostomy and use of steroids or chemotherapy were found to be the most important risk factors for mortality in ICU $[29,30]$ similar to our study and those of others [31,32]. Although, the clinical significance of Methicillin-resistance has been questioned in the past there is now a widespread acknowledgement of the pathogenicity of MRSA. It has emerged as a significant cause of both nosocomial and community acquired infections. Furthermore, during the past decade there has been a steady increase in the incidence of infections caused by this bacterium [33].

Many investigators have reported an increase in the incidence of MRSA during recent years, most of which originated from wounds (pus) [34]. We also found a high rate of MRSA isolates i.e. $35.38 \%$ from the clinical specimens also showed multiple drug resistance. In our study (64.62\%) MSSA (Methicillin sensitive Staphylo- coccus aureus) isolates were resistant most of the antibiotics tested and $1.53 \%$ of the isolates were VRSA. Prevention of MRSA infections merit discussion as once introduced in a hospital MRSA is very difficult to eradicate [35]. After introduction within hospital, MRSA spreads rapidly by hands of medical personnel. Colonized employees of the hospital such as asymptomatic nasal carriers and infected patients acting as reservoirs are important sources in the spread of this organism [36]. Multiple, prolonged use of antibiotics and prolonged hospitalization are other important factors which make hospitals an ideal place for transmission and perpetuation of MRSA.

Resistant strains are not only a major obstacle in treatment, but once established, infections are also likely to ratchet up the possibility of further transmission. In 1982, an American hospital outbreak reported an incidence of $30 \%$ MRSA [37]. Other studies conducted in Japan reported an incidence of about $41.5 \%$ by 1992 [38]. There are many reports of increasing resistance of $S$. aureus from our country [39]. In order to reduce the problem of antibiotic resistance, it is mandatory to survey and screen all clinical isolates for resistance. Our efforts should be concentrated not only on antibiotic use, but also on other confounding factors that contribute to resistance such as infection control practices. Contact isolation and strict asepsis should be enforced. This area warrants further studies on a larger number of isolates from various parts of the country in order to develop and apply evidence based guidelines on countering resistance [40].

\section{Conclusion}

Therefore, regular surveillance of hospital-associated infections including monitoring of antimicrobial (especially Vancomycin and other newer glycopeptides) susceptibility pattern of MRSA and formulation of a definite antimicrobial policy may be helpful for reducing the incidence of these infections. Infected or colonized patients may be isolated in a single room or isolation unit to prevent the spread of MRSA. Knowledge about MRSA and carrier status needs to be raised among the health staff of the hospital and control measures need to be implemented consistently in order to reduce the burden of MRSA infection in the hospital environment. A further study of MRSA may be done for the epidemiological mapping of these infections.

\section{Acknowledgements}

The authors are grateful to the Post Graduate Department of Studies in Microbiology and Biotechnology, Karnatak University Dharwad for providing the necessary facilities. 


\section{REFERENCES}

[1] D. E. Townsend, N. Ashdown, S. Bolton, J. Bradley, G. Duckworth, E. C. Moorhouse, et al., "The International Spread of Methicillin-Resistant Staphylococcus aureus," Journal of Hospital Infection, Vol. 9, No. 1, 1987, pp. 6071. doi:10.1016/0195-6701(87)90097-1

[2] A. Voss, D. Milatovic, C. Wallrauch-Schwarz, V. T. Rosdahl and I. Braveny, "Methicillin-Resistant Staphylococcus aureus in Europe," European Journal of Clinical Microbiology \& Infectious Diseases, Vol. 13, No. 1, 1994, pp. 50-55. doi:10.1007/BF02026127

[3] R. P. Wenzel, R. L. Thompson and S. M. Landry, "Hospital Acquired Infections in Intensive Care Unit Patients: An Overview with Emphasis on Epidemics," American Journal of Infection Control, Vol. 4, No. 5, 1983, pp. 371-375.

[4] J. D. Edgeworth, D. F. Treacher and S. J. Eykyn, “A 25Year Study of Nosocomial Bacteremia in Adult Intensive Care Unit," Critical Care Medicine, Vol. 27, No. 8, 1999 , pp. 1421-1428. doi:10.1097/00003246-199908000-00002

[5] J. L. Vincent, D. J. Bihari, P. M. Suter, et al., "The Prevalence of Nosocomial Infection in Intensive Care Units in Europe: Results of the European Prevalence of Infection in Intensive Care (EPIC) Study," The Journal of the American Medical Association, Vol. 274, No. 8, 1995, pp. 639644. doi:10.1001/jama.1995.03530080055041

[6] R. P. Wenzel and M. B. Edmond, "The Impact of Hospital-Acquired Bloodstream Infections," Emerging Infectious Disease, Vol. 7, No. 2, 2001, pp. 174-177. doi:10.3201/eid0702.010203

[7] J. E. Peacock Jr., F. J. Marsik and R. P. Wenzel, "Methicillin-Resistant Staphylococcus aureus: Introduction and Spread within a Hospital," Annals of Internal Medicine, Vol. 93, No. 4, 1980, pp. 526-532.

[8] J. M. Hughes, "Setting Priorities: Nationwide Nosocomial Infection Prevention and Control Programs in the USA," European Journal of Clinical Microbiology \& Infectious, Vol. 6, No. 3, 1987, pp. 348-351. doi:10.1007/BF02017638

[9] S. Al-Obeid, E. Collatz and L. Gutman, "Mechanism of Resistance to Vancomycin in Enterococcus faecium D366 and Enterococcus fecalis A256," Antimicrobial Agents and Chemotherapy, Vol. 34, No. 2, 1990, pp. 252-256.

[10] S. Salmenlinna, O. Lyytikainen and J. Vuopio-Varkila, "Community Acquired methicillin resistant Staphylococcus aureus, Finland," Emerging Infectious Diseases, Vol. 18, No. 1, 2002, pp. 602-607.

[11] World Health Organization, "Recommendations for the Control of Methicillin Resistant Staphylococcus aureus (MRSA)," WHO, Geneva, 1996.

[12] E. Dickinson, "Mortality from Methicillin-Resistant Staphylococcus aureus in England and Wales: Analysis of Death Certificates," British Medical Journal, Vol. 325, No. 7377, 2002, pp. 1390-1391. doi:10.1136/bmj.325.7377.1390

[13] L. Collier, A. Balows and M. Sussman, "Bacterial Infections. Topley and Wilson's Microbiology and Microbial Infections," 9th Edition, Arnold Publication, London, 1998, pp. 231-256.

[14] B. N. Doebbeling, "The Epidemiology of Methicillin Resistant Staphylococcus aureus Colonization and Infection," Journal of Chemotherapeutics, Vol. 7, Suppl. 3, 1995, pp. 99-103.

[15] A. L. Panlilio, D. H. Culver, R. P. Gaynes, S. Banerjee, T. S. Henderson, J. S. Tolson, et al., "Methicillin-Resistant Staphylococcus aureus in US Hospitals, 1975-1991," Infection Control and Hospital Epidemiology, Vol. 13, No. 10, 1992, pp. 582-586. doi:10.1086/646432

[16] S. Verma, S. Joshi, V. Chitnis, N. Hemwani and D. Chitnis, "Growing Problem of Methicillin Resistant Staphylococci-Indian Scenario," Indian Journal of Medical Sciences, Vol. 54, No. 12, 2000, pp. 535-40.

[17] A. P. Mehta, C. Rodrigues, K. Sheth, S. Jani, A. Hakimiyar and N. Fazalbhoy, "Control of Methicillin Resistant Staphylococcus aureus in a Tertiary Care Centre: A Five Year Study," Indian Journal of Medical Microbiology, Vol. 16, No. 1, 1998, pp. 31-34.

[18] K. Hiramatsu, H. Hanaki and T. Ino, "Methicillin-Resistant Staphylococcus aureus Clinical Strain with Reduced Vancomycin Susceptibility," Journal of Antimicrobial Chemotherapy, Vol. 40, No. 1, 1997, 135-136. doi:10.1093/jac/40.1.135

[19] J. E. Geraci, "Some Laboratory and Clinical Experiences with a New Antibiotic, Vancomycin," In: Antibiotic Annual 1955-1956, Medical Encyclopedia, New York, 1956, pp. 90-106.

[20] R. C. Moellering, "The Specter of Glycopeptide Resistance: Current Trends and Future Considerations," American Journal of Medicine, Vol. 104, Suppl. 5A, 1998, pp. 3S-6S. doi:10.1016/S0002-9343(98)00148-X

[21] C. U. Tuazon and H. Miller, "Clinical and Microbiologic Aspects of Serious Infections Caused by Staphylococcus epidermidis," Scandinavian Journal of Infectious Diseases, Vol. 15, No. 4, 1983, pp. 347-360.

[22] C. G. Unakal and B. B. Kaliwal, "Vancomycin-Resistant Staphylococcus aureus Containing van A Gene Isolated from Clinical Samples of Community Health Care Centers of North Karnataka, India," Proceedings of International Society BioTechnology Conference (ISBT-2008), Gangtok, 28-30 December 2008, pp. 464-468.

[23] National Committee for Clinical Laboratory Standards, "Methods for Dilution a Antimicrobial Susceptibility Tests for Bacteria That Grow Aerobically: Approved Standard 2000, M7-A5," 5th Edition, NCCLS, Wayne, 2000.

[24] J Valles, C Leon and F Alvarez-Lerma, "Nosocomial Bacteremia in Critically Ill Patients: A Multi-Center Study Evaluating Epidemiology and Prognosis," Clinical Infectious Diseases, Vol. 24, No. 3, 1997, pp. 387-395. doi:10.1093/clinids/24.3.387

[25] D. R. Schaberg, D. H. Culver and R. P. Gaynes, "Major Trends in the Microbial Etiology of Nosocomial Infection," American Journal of Medicine, Vol. 91, Suppl. 3B, 1991, pp. 72-75. doi:10.1016/0002-9343(91)90346-Y

[26] W. Sligl, G. Taylor and P. G. Brindley, "Five Years of Nosocomial Gram-Negative Bacteremia in a General Intensive Care Unit: Epidemiology, Antimicrobial Suscepti- 
bility Patterns, and Outcomes," International Journal of Infectious Diseases, Vol. 10, No. 4, 2006, pp. 320-325. doi:10.1016/j.ijid.2005.07.003

[27] V. Suljagic, M. Cobeljic, S. Jancovic, V. Mirovic, L. Markovic-Denic, P. Romic and D. Mikic, "Nosocomial Bloodstream Infections in ICU and Non-ICU Patients," American Journal of Infection Control, Vol. 33, No. 6, 2005, pp. 333-340. doi:10.1016/j.ajic.2005.03.010

[28] T. N. Jang, B. I. Kuo, S. H. Shen, C. P. Fung, S. H. Lee, T. L. Yang and C. S. Huang, "Nosocomial Gram-Negative Bacteremia in Critically Ill Patients: Epidemiologic Characteristics and Prognostic Factors in 147 Episodes," Journal of the Formosan Medical Association, Vol. 98, No. 7, 1999, pp. 465-473.

[29] A. Colpan, E. Akinci, A. Erbay, N. Balaban and H. Bodur, "Evaluation of Risk Factors for Mortality in Intensive Care Units: A Prospective Study from a Referral Hospital in Turkey," American Journal of Infection Control, Vol. 33, No. 1, 2005, pp. 42-47. doi:10.1016/j.ajic.2004.09.005

[30] K. B. Laupland, D. A. Zygun, C. J. Doig, S. M. Bagshaw, L. W. Svenson and G. H. Fick, "One-Year Mortality of Bloodstream Infection-Associated Sepsis and Septic Shock among patients Presenting to a Regional Critical Care System," Intensive Care Medicine, Vol. 31, No. 2, 2005 , pp. 213-219. doi:10.1007/s00134-004-2544-6

[31] M. Arvanitidou, E. Katikaridou, J. Douboyas and A. Tsakris, "Prognostic Factors for Nosocomial Bacteraemia Outcome: A Prospective Study in a Greek Teaching Hospital," Journal of Hospital Infection, Vol. 61, No. 3, 2005, pp. 219-324. doi:10.1016/j.jhin.2005.03.006

[32] Y. C. Lin, T. L. Chen, H. L. Ju, H. S. Chen, F. D. Wang, K. W. Yu and C. Y. Liu, "Clinical Characteristics and Risk Factors for Attributable Mortality in Enterobacter cloacae Bacteremia," Journal of Microbiology, Immunology and Infection, Vol. 39, No. 1, 2006, pp. 67-72.
[33] A. Alborzi, B. A. Pourabbas, H. Salehi, B. H. Pourabbas, B. Oboodi and M. R. Panjehshahin, "Prevalence and Pattern of Antibiotic Sensitivity of Methicillin Sensitive and Resistant Staphylococcus aureus in Shiraz-Iran," Iranian Journal of Medical Sciences, Vol. 25, 2000, pp. 1-8.

[34] S. Vidhani, P. L. Mehndiratta and M. D. Mathur, "Study of Methicillin Resistant S. aureus (MRSA) Ilolates from High Risk Patients," Indian Journal of Medical Microbiology, Vol. 19, 2001, pp. 13-16.

[35] J. P. Arbuthnott, D. C. Coleman and J. S. DeAsavedo, "Staphylococcal Toxins in Human Disease," Journal of Applied Bacteriology, Vol. 69, Suppl. 19, 1990, pp. 101S107S. doi:10.1111/j.1365-2672.1990.tb01802.x

[36] R. L. Thompson, I. Cabezuclo and R. P. Werizel, "Epidemiology of Nosocomial Infections Caused by Methicillin-Resistant Staphylococcus Aureus Infections Caused by Methicillin-Resistant Staphylococcus Aureus," Annals of Internal Medicine, Vol. 97, No. 3, 1982, pp. 309-317.

[37] J. R. Myers and C. C. Linnemann, "Bacteremia Due to Methicillin Resistant Staphylococcus aureus," Journal of Infectious Diseases, Vol. 145, No. 4, 1982, pp. 532-536. doi:10.1093/infdis/145.4.532

[38] S. Nishijima, S. Namura, K. Mitsuya, et al., "The Incidence of Isolation of MRSA Strains from Skin Infections during 1989-1991," Journal of Dermatology, Vol. 20, No. 4, 1993, pp. 193-197.

[39] S. K. Mathur, S. Singhal, K. N. Prasad, et al., "Prevalence of Methicillin Resistant Staphylococcus aureus (MRSA) in a Tertiary Care Hospital," Indian Journal of Medical Microbiology, Vol. 12, 1994, pp. 96-101.

[40] K. G. Bhat and M. G. Bhat, "Prevalence of Nosocomial Infections Due to Methicillin Resistant Staphylococcus aureus in Mangalore, India," Biomedicine, Vol. 17, No. 1, 1997, pp. 17-20. 